\title{
Effect of fiber fatigue rupture on monotonic and cyclic crack bridging laws in discontinuous fiber reinforced composites 短緎維補強複合材料の単調及び綝返し載荷下ひび割れ架橋則 における繊維疲労破断の影響
}

\author{
Takashi Matsumoto* \\ 松本 高志 \\ * Member, Ph.D., Assoc. Prof., Division of Built Environment, Hokkaido University (Kita-13, Nishi-8, Kita-ku, Sapporo 060-8628)
}

\begin{abstract}
This paper presents the micromechanical modeling of fiber bridging constitutive laws of a discontinuous fiber reinforced composite (DFRC) under monotonic and cyclic loading with the effects of fiber fatigue rupture. Based on the considerations of fatigue damage on randomly distributed fibers under the fatigue loading of constant crack opening amplitude, the monotonic and cyclic fiber bridging constitutive laws are derived. It is shown that, as fiber fatigue rupture proceeds, maximum bridging stress as well as crack opening displacement at which bridging stress vanishes are decreased. Also, the critical normalized number of cycles, $n_{\text {crit }}^{*}$ at which crack bridging degradation takes place, is analytically derived, and its role is explained for the design of a DFRC against fatigue.
\end{abstract}

Key Words: Bridging law, discontimous fiber reinforced composite, cyclic loading, fiber fatigue rupture

\section{Introduction}

Recently, short fiber reinforced cementitious composites are increasingly applied into civil engineering structures. These composites are placed in structural members in such a way that their high strength, ductility, and fracture toughness in tension are utilized in order to increase the safety, ductility, and durability of structures. It is known that those beneficial composite properties are thanks to bridging fibers that transfer stresses across a crack, and it is important to understand a fiber bridging constitutive law, which is the relation between bridging stress and crack opening displacement, based on micromechanical parameters such as fiber length, fiber diameter, fiber modulus, interfacial frictional bond strength, and so on.

Some of the structural applications show that the composites are used in structural members where severe load repetitions are present, and that the composites are expected to yield long-term durabilities with their high fatigue strength, high crack resistance, and crack width control ability. In order to evaluate the long-term durabilities under load repetitions, it is necessary to understand a fiber bridging constitutive law under cyclic loading and also understand the long-term degradation of fiber bridging itself. The fiber bridging constitutive law under cyclic loading has been derived already, and it has shown the validity in comparison to experimental measurements ${ }^{1)}$. The next step is the consideration of the long-term degradation in fiber bridging itself. This is because fiber bridging itself can be considered to degrade in the long run, namely the transferred stresses decay as load repetitions continue. For degradation, there can be two possible mechanisms. One is interfacial bond degradation, and the other is fiber fatigue rupture.

Under cyclic loading, there is ample evidence to show that interfacial frictional bond strength changes under a large number of cyclic loads, e.g. in aligned continuous fiber reinforced ceramic matrix composites such as $\mathrm{SiC} / \mathrm{CAS}^{2 / 34) 556)}$ and $\mathrm{SiC} / \mathrm{SiC}^{788}$ and in steel fiber reinforced concrete ${ }^{9)}$ The interfacial bond degradation has been implemented into the bridging constitutive law in a fatigue crack growth analysis, and an experimental crack growth measurement was successfully reproduced $^{10)}$. Furthermore, the stress-life (S-N) relation of 
various kinds of fiber reinforced concrete were reproduced with this analysis ${ }^{1112}$.

On the other hand, for fiber fatigue rupture under cyclic loading, there are fewer evidences.

Aligned continuous $\mathrm{SiC}$ fibers in a titanium alloy were observed to fracture under tensile fatigue loading ${ }^{13}$. Crack growth behavior of this fiber composite is significantly influenced by the fiber fracture, namely, the fiber fracture coincides with the onset of final accelerated crack growth. This study shows that the fiber fatigue rupture leads to the degradation of crack bridging stress and in turn to the final failure.

The presence of fiber fatigue rupture can be readily proven by the comparison of crack surfaces of static and fatigue specimens ${ }^{1415) 16}$.

Randomly distributed discontinuous polyethylene fibers in a cement matrix exhibited severe fatigue rupture in fatigue test ${ }^{14)}$. Close-up pictures of the crack surfaces of static and fatigue specimens are clearly different in terms of fiber protrusion length. The static specimen showed that fibers were pulled out with no rupture as the crack opened up. On the other hand, the fatigue specimen showed that fibers were gradually ruptured during fatigue loading and that almost all the fibers were ruptured by the final failure of the specimen. This comparison reveals the susceptibility of the polyethylene fibers to fatigue damage, because the fibers are gradually ruptured during fatigue loading, although the length of fibers is designed so that no fiber rupture can take place during static loading (i.e. the fiber length is less than the critical length based on fiber diameter, strength and interface friction consideration).

Similarly, polyvinyl alcohol fibers in a cement matrix suffer from fiber rupture more in the fatigue specimens than in the static specimens ${ }^{1516)}$. However, it should be noted that this composite is designed so that fiber rupture is allowed during static loading.

Therefore, having observed these evidences of fiber fatigue rupture, it is necessary to evaluate the effect of fiber fatigue rupture on monotonic and cyclic crack bridging laws in discontinuous fiber reinforced composites. The evaluation will be done by constructing a micromechanical model of the fiber bridging constitutive laws.

This paper addresses on the fatigue damage on fibers. Progressive fiber fatigue rupture during the fatigue loading of constant crack opening displacement amplitude is taken into account, and the analytical expressions of fiber bridging stress crack opening displacement $\left(\sigma_{f}-\delta\right)$ and fiber bridging stress amplitude - crack opening displacement amplitude $\left(\Delta \sigma_{f}-\Delta \delta\right)$ relation with fiber fatigue rupture are obtained.

\section{Review of Single Fiber and Composite Behavior under Monotonic and Cyclic Loading}

Before the derivation of $\sigma_{f^{-}} \delta$ and $\Delta \sigma_{f^{-}}-\Delta \delta$ relations with

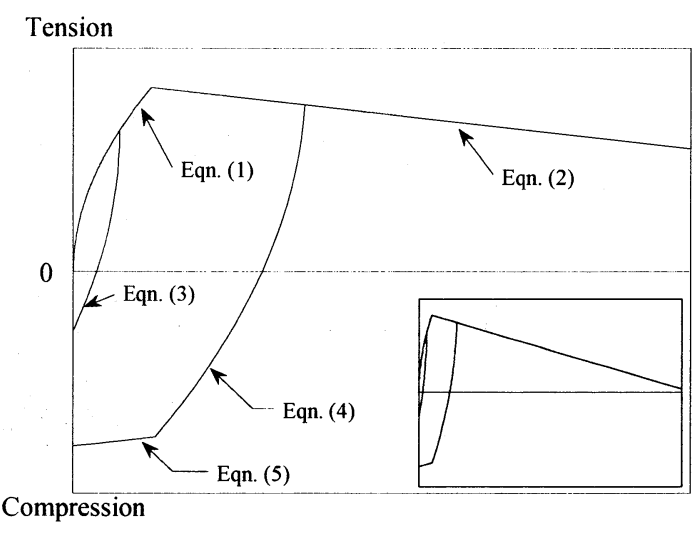

Crack Opening Displacement, $\delta$

Fig. 1 Single fiber loading-unloading curves with corresponding equations (insert for complete curves) ${ }^{17}$

fiber fatigue rupture, a brief review will be done in this section $^{117118)}$. Since fiber bridging stress is a sum of the pull-out loads carried by individual fibers, single fiber pull-out load - crack opening displacement $(P-\delta)$ relation is reviewed ${ }^{17 / 18}$ (see Fig. 1).

The $P$ - $\delta$ relation during debonding stage is given by

$$
P_{d}(\delta)=\frac{\pi}{2} \sqrt{E_{f} d_{f}^{3} \tau \delta} \quad \text { for } \delta \leq \delta_{o}
$$

where $E_{f}=$ fiber modulus, $d_{f}=$ fiber diameter, $\tau=$ interfacial frictional bond strength, $\delta_{o}=\left(4 \tau l^{2}\right) /\left(E_{f} d_{f}\right), l=$ embedment length, and during sliding stage by

$$
P_{s l}(\delta)=\pi \tau d_{f}(l-\delta) \quad \text { for } \delta_{o}<\delta \leq l .
$$

Under cyclic loading, pull-out load amplitude - crack opening displacement amplitude $(\Delta P-\Delta \delta)$ relation is given as follows ${ }^{1)}$.

For fihers that have heen in dehonding stage under the preceding pull-out loading, we have:

$$
\Delta P_{1}(\Delta \delta)=\pi \sqrt{\frac{\tau d_{f}^{3} E_{f} \Delta \delta}{2}}
$$

and for fibers that have been in sliding stage under the preceding pull-out loading, the fibers undergo unstretching and contracting according to

$$
\Delta P_{2}(\Delta \delta)=\Delta P_{1}(\Delta \delta)=\pi \sqrt{\frac{\tau d_{f}^{3} E_{f} \Delta \delta}{2}}
$$

Furthermore, when these fibers slide back into the matrix after unstretching and contracting, the $\Delta P-\Delta \delta$ relation is given by

$$
\Delta P_{3}\left(\Delta \delta, \delta_{\max }\right)=\pi \tau d_{f} \Delta \delta+2 P_{\max }
$$

where $\delta_{\max }=$ crack opening displacement at which unloading 
starts and $P_{\max }=\pi \tau d_{f}\left(l-\delta_{\max }\right)$.

Based on the above single fiber behavior $(P-\delta$ and $\Delta P-\Delta \delta$ relations), the fiber bridging constitutive laws under monotonic and cyclic loading were derived. The details of the laws can be found elsewhere ${ }^{1)}$. Here, only the definitions of parameters are introduced for the later use. Fig. 2 shows the bridging stress crack opening displacement behavior of a cracked composite. Under monotonic loading, the bridging stress increases with crack opening displacement until it reaches a peak value, $\sigma_{o}$, at the corresponding crack opening displacement, $\delta^{*}$, and it decays to zero together with fiber pull-out. Here, $\sigma_{o}=V_{f} \tau\left(L_{f} / d_{f}\right) / 2$, where $V_{f}=$ fiber volume fraction and $L_{f}=$ fiber length, and $\delta^{*}$ corresponds to the maximum value of $\delta_{o}$ with $l=L_{f} / 2$, at which all fibers have completed debonding. For cyclic loading, we consider unloading and reloading. The unloading starts from a point on the monotonic relation, and the coordinates of the point are denoted by $\sigma_{\max }$ and $\delta_{\max }$. The fiber bridging constitutive law is defined with this point the origin. Namely, bridging stress amplitude, $\Delta \sigma$, and crack opening displacement amplitude, $\Delta \delta$, are measured from this point, as shown in Fig. 2. Therefore, the normalized fiber bridging constitutive laws will be expressed in terms of $\alpha=\delta_{\max } / \delta^{*}$ and $\beta=\Delta \delta / \delta_{\max }$.

\section{Fatigue Damage on Randomly Distributed Fibers}

It is assumed that fiber fatigue rupture takes place on a deterministic basis according to the stress-life (S-N) relation of a fiber (Fig. 3) and that surviving fibers determined in this manner exert fiber bridging stress across a crack under either monotonic or cyclic loading. Here, $\Delta \sigma_{s f}$ is stress amplitude in a single fiber, $\sigma_{f u}$ is fiber ultimate strength, $n_{f}$ is cycles to failure, and $b$ is a coefficient. The derivation of the constitutive laws with the effect of fiber fatigue rupture is summarized below, and details of the derivation can be found in Appendix.

Under cyclic loading of a given fiber composite, each of fibers is subjected to cyclic pull-out/push-in loading. The amplitude and magnitude of the resulting single fiber load depend not only on the overall load amplitude and magnitude, but also on the fiber location and orientation at a designated crack plane, and, in turn, the fatigue life of each fiber is dependent on these initial conditions and the number of cycles applied. From the viewpoint of each cyclically loaded fiber, there can be two loading conditions: with or without load redistribution among fibers.

Load redistribution takes place among fibers, when the lost load due to gradually fatigue ruptured fibers has to be taken by surviving fibers under the same or larger overall load. For example, this is the case when a bridged crack with uniform crack profile is cyclically loaded under constant load amplitude or when a bridged crack with non-uniform crack profile grows under constant cyclic load amplitude.

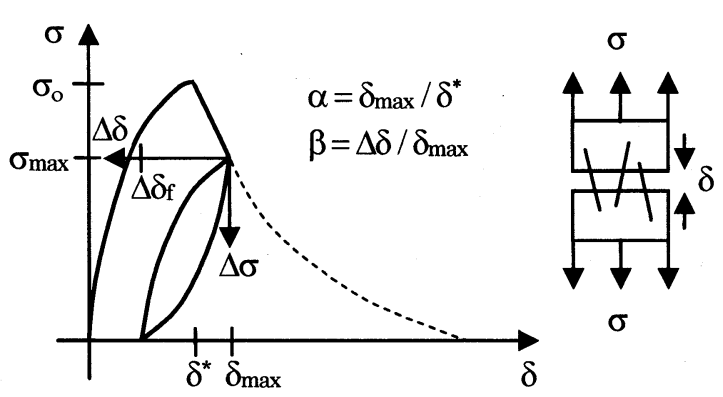

Fig. 2 Definitions of parameters

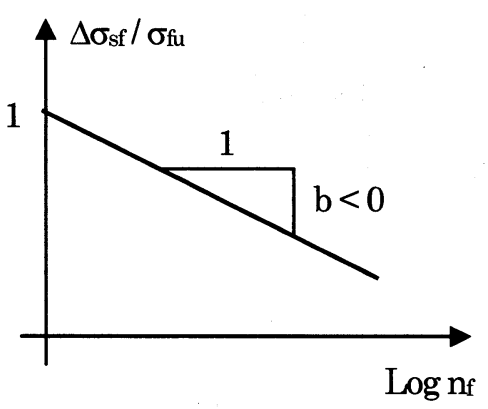

Fig. 3 Assumed stress-life (S-N) relation of a fiber

On the other hand, no load redistribution happens when a fiber composite cyclically loaded under the condition of constant crack opening displacement amplitude of a uniform profile crack. Comparing these two loading conditions (with or without load redistribution), fatigue damage on fibers is certainly more severe with load redistribution, since surviving fibers have to sustain increasing load amplitude as fibers are gradually lost due to fatigue rupture. In this study, we will focus on the case of no load redistribution (constant crack opening displacement amplitude). This is because the problem can be simplified, but it provides fundamental insights and a basis even for the case of load redistribution.

First, we will consider the group of remaining fibers under monotonic and cyclic loading with or without fiber fatigue rupture. For the fiber location, $z$, and orientation, $\phi$ (Fig. 4), 3-D uniform randomness is assumed, and, before any loading happens, all the fibers are included in the domain of fiber location $\left(z /\left(\left(L_{f} / 2\right) \cos \phi\right)\right) \in(0,1)$, hereafter $\left.z /\left(\left(L_{f} / 2\right) \cos \phi\right)\right)$ is denoted as $w)$ and orientation $(\phi \in(0, \pi / 2))$ shown in Fig. 5. When monotonic loading takes place and the crack is opened up to $\widetilde{\delta}_{\max }=\delta_{\max } /\left(L_{f} / 2\right)$, the domain of remaining fibers is reduced $\left(w \in\left(0,1-\widetilde{\delta}_{\max }\right)\right)$ due to the pull-out of fibers which starts from those with short embedment length, $l$ (large $w$, note that $l=L_{f} / 2-z / \cos \phi$ (Fig. 4)). The domain of remaining fibers is divided into two: fibers in debonding $\left(w \in\left(0, z_{o}\right)\right)$ and sliding ( $w \in\left(z_{o}, 1-\widetilde{\delta}_{\max }\right)$ ), and fiber bridging stress under monotonic loading can be obtained by integrating (1) and (2) in the two divided domains respectively. Upon unloading of this 
loaded fiber composite, the domain is not reduced, but divided into three with respect to $w$ (Fig. 5). The fibers that have been in debonding $\left(w \in\left(0, z_{o}\right)\right)$ in the preceding monotonic loading are unloaded according to (3), and the fibers that have been in sliding ( $w \in\left(z_{o}, 1-\widetilde{\delta}_{\max }\right)$ ) undergo unstretching and contracting (4), followed by sliding-in (5). The subdomain for the fibers that have been in sliding is now divided into two by

$$
z_{1}=\frac{\Delta \widetilde{\delta}}{2}+1-\widetilde{\delta}_{\max }-\sqrt{\frac{\Delta \widetilde{\delta}}{2 \widetilde{\delta}^{*}}}
$$
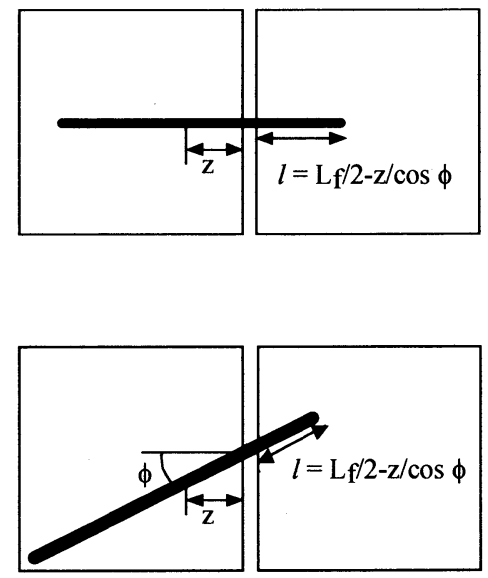

Fig. 4 Fiber centroidal distance, $z$, and orientation, $\phi$

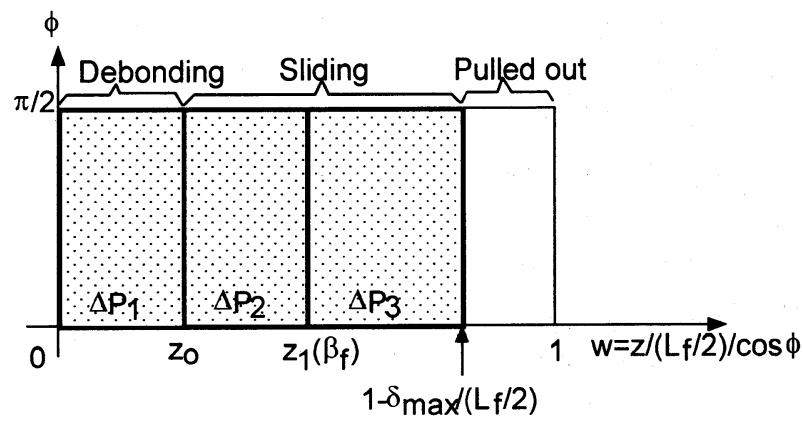

Fig. 5 Domain of the fiber centroidal distance, $\mathrm{z}$, and orientation, $\phi$

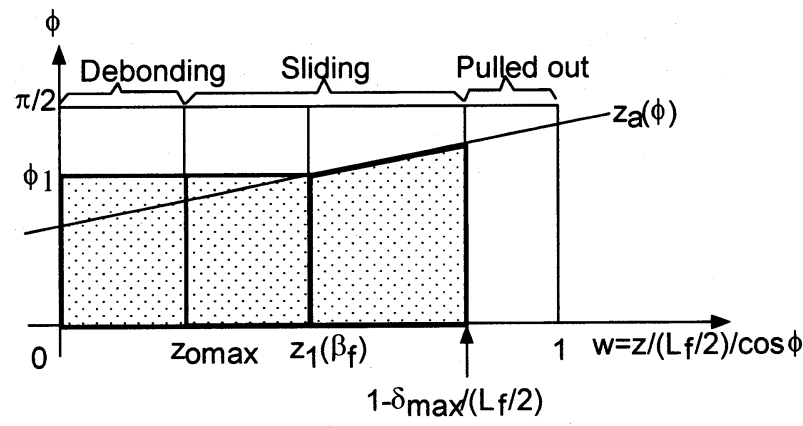

Fig. 6 Reduced domain of the fiber centroidal distance, $z$, and orientation, $\phi$, due to fiber fatigue rupture
Fiber bridging stress under cyclic loading can be obtained by integrating (3), (4), and (5) in the three divided domains respectively, and thus it is dependent on $\widetilde{\delta}_{\max }$.

As fatigue loading proceeds, the domain is gradually reduced by fiber fatigue rupture, resulting in degradation of fiber bridging stress under either monotonic or cyclic loading. In this study, it is assumed that fibers are ruptured not under monotonic loading, but under fatigue loading, so the domain is reduced in such a way that fatigue ruptured fibers are discounted according to the S-N relation of a fiber. The $\mathrm{S}-\mathrm{N}$ relation of a fiber is assumed to be

$$
\frac{\Delta \sigma_{s f}}{\sigma_{f u}}=n_{f}^{b}=\frac{1}{n_{f}^{c}}
$$

(see Fig. 3). This simple assumption does not account for fatigue limit, below which fatigue failure never occurs, so every fiber fails in fatigue at a finite number of cycles. It also does not account for mean stress effect, which changes the coefficient, $b$, of the S-N relation under different mean stress of a load cycle. According to the assumed S-N relation of a fiber, the condition for fibers to have survived for $n$ cycles of fatigue loading is given by

$$
n<n_{f}=\left(\frac{\sigma_{f u}}{\Delta \sigma_{s f}}\right)^{\frac{1}{c}}
$$

The stress amplitude in a single fiber, $\Delta \sigma_{s,}$, can be obtained through dividing the fiber pull-out/push-in load by the fiber cross sectional area:

$$
\Delta \sigma_{s f}=\frac{\Delta P e^{f \phi}}{\pi\left(d_{f} / 2\right)^{2}}
$$

where $\Delta P=$ fiber pull-out/push-in load amplitude applied for $n$ cycles and is either $\Delta P_{1}, \Delta P_{2}$, or $\Delta P_{3}$ depending on the embedment length of a fiber (Fig. 5). The factor $e^{f \phi}$ refers to a snubbing effect which describes the mechanical interactions between a loaded inclined fiber and the matrix material ${ }^{19}$. It is assumed that the $\Delta P-\Delta \delta$ relation is approximated in the same way by Matsumoto ${ }^{1)}$. From (8) and (9), the survival condition of a fiber after $n$ cycles becomes

$$
n<n_{f}=\left(\frac{\sigma_{f u}}{\left(\Delta P e^{f \phi}\right) /\left(\pi d_{f}^{2} / 4\right)}\right)^{\frac{1}{c}}
$$

First, fibers within $w=0 \sim z_{o}$ are loaded according to $\Delta P=\Delta P_{1}$, then from (10) we have

$$
\phi<\frac{1}{f} \ln \left[\frac{1}{n^{c}} \frac{L_{c}}{L_{f}}\left(\sqrt{\frac{1}{2}\left(\frac{\Delta \widetilde{\delta}_{f}}{\widetilde{\delta}^{*}}\right)}\right)^{-1}\right]=\phi_{1}
$$

where $L_{c}=\left(\sigma_{f u} d_{f}\right) /(4 \tau), \Delta \widetilde{\delta}_{f}=\Delta \delta_{f} /\left(L_{f} / 2\right)=$ normalized 
crack opening displacement amplitude applied for $n$ cycles, $\widetilde{\delta}^{*}$ $=\delta^{*} /\left(L_{f} / 2\right)=\left(2 \tau L_{f}\right) /\left(E_{f} d_{f}\right)$. Altematively, in a normalized form, we have

$$
\phi<\frac{1}{f} \ln \left[\frac{1}{n^{*}}\left(\sqrt{\frac{\alpha_{f} \beta_{f}}{2}}\right)^{-1}\right]=\phi_{1}
$$

where $n^{*}=\left(n^{c} L_{f}\right) / L_{c}, \alpha_{f}=\widetilde{\delta}_{\max } / \widetilde{\delta}^{*}$, and $\beta_{f}=\Delta \widetilde{\delta}_{f} / \widetilde{\delta}_{\max }$. Therefore, the domain of surviving fibers is reduced, and only the fibers with $w=0 \sim z_{o}$ and $\phi=0 \sim \phi_{1}$ can survive for $n$ cycles under the normalized constant crack opening displacement amplitude of $\Delta \widetilde{\delta}_{f}$ (Fig. 6). Second, fibers within $w=z_{o} \sim z_{1}$ are also loaded according to $\Delta P=\Delta P_{1}$, since it is assumed that $\Delta P_{2}=\Delta P_{1}$. Hence the domain of surviving fibers is again limited by $\phi_{1}$, and only the fibers with $w=0 \sim z_{o}$ and $\phi=0 \sim \phi_{1}$ can survive (Fig. 6). Finally, fibers are loaded according to $\Delta P$ $=\Delta P_{3}$, when they belong to $w=z_{1} \sim 1-\widetilde{\delta}_{\max }$. Here, from (10), we have

$$
w \geq \frac{\Delta \widetilde{\delta}_{f}}{2}+1-\widetilde{\delta}_{\max }-\frac{L_{c}}{L_{f}} \frac{1}{n^{c} e^{f \phi}}=z_{a}(\phi)
$$

or, in a normalized form,

$$
w \geq \frac{\alpha_{f} \beta_{f} \tilde{\delta}^{*}}{2}+1-\alpha_{f} \tilde{\delta}^{*}-\frac{1}{n^{*} e^{f \phi}}=z_{a}(\phi)
$$

Therefore, the domain of surviving fibers is limited by (13) or (14), and only the fibers that satisfy $w=z_{1} \sim 1-\widetilde{\delta}_{\max }, \phi \geq 0$, and $w \geq z_{d}(\phi)$ can survive (Fig. 6). In the case of $\Delta P=\Delta P_{3}$, the limit is dependent also on $\widetilde{\delta}_{\max }$.

Based on the domain of the surviving fibers after $n$ cycles of constant crack opening displacement amplitude loading with $\widetilde{\delta}_{\max }$ and $\Delta \widetilde{\delta}_{f}$, we can construct the monotonic and cyclic fiber bridging constitutive laws. Details of the derivation can be found in Appendix.

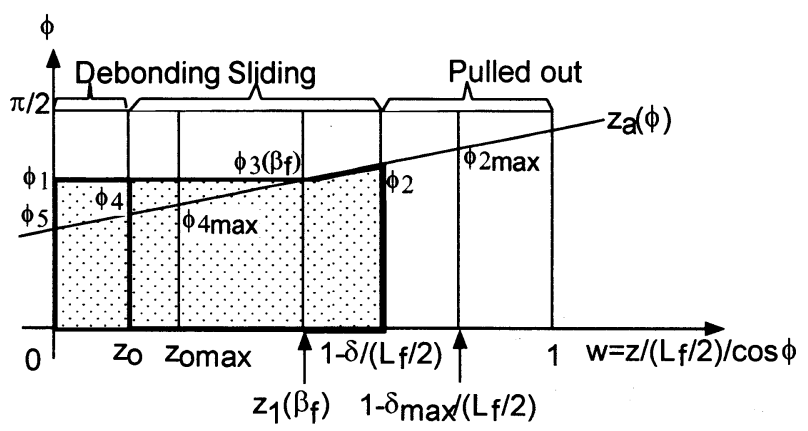

Fig. 7 Domain of the fiber centroidal distance, $\mathrm{z}$, and orientation, $\phi$, for monotonic bridging constitutive law after fiber fatigue rupture

\section{Monotonic Constitutive Law with Fiber Fatigue Rupture}

The monotonic fiber bridging constitutive law with the effect of fiber fatigue rupture is given below, and the domain of surviving fibers is shown in Fig. 7 (see Appendix for details).

When a fiber composite is fatigue-loaded for $n$ cycles in the prepeak region $\left(\alpha_{f}=\widetilde{\delta}_{\max } / \widetilde{\delta}^{*}<1\right)$ and afterwards it is further loaded under monotonic loading, the monotonic constitutive law for the prepeak $\left(\alpha_{f}<\alpha=\widetilde{\delta} / \widetilde{\delta}^{*}<1\right)$ is given by, for $\alpha<(1-$ $\left.z_{1}(\beta)\right) / \widetilde{\delta}^{*}$,

$$
\begin{aligned}
& \left.\tilde{\sigma}_{f}\right|_{\text {prepeak }}=g_{1}\left(\phi_{1}\right) 2 \sqrt{\alpha}(1-\alpha) \\
& +g_{1}\left(\phi_{1}\right)\left(\left(\alpha \widetilde{\delta}^{*}\right)^{2}-2 \alpha \widetilde{\delta}^{*} \sqrt{\alpha}+\alpha\right) \\
& +g_{2}\left(f, \phi_{1}, \phi_{2}\right)\left(\left(1-\alpha \widetilde{\delta}^{*}\right)-\left(1-\alpha_{f} \widetilde{\delta}^{*}\right)-\frac{1}{2} \alpha_{f} \beta_{f} \widetilde{\delta}^{*}\right)^{2} \\
& +g_{2}\left(0, \phi_{1}, \phi_{2}\right) 2\left(\left(1-\alpha \widetilde{\delta}^{*}\right)-\left(1-\alpha_{f} \widetilde{\delta}^{*}\right)-\frac{1}{2} \alpha_{f} \beta_{f} \widetilde{\delta}^{*}\right) \frac{1}{n^{*}} \\
& +g_{2}\left(-f, \phi_{1}, \phi_{2}\right)\left(\frac{1}{n^{*}}\right)^{2}
\end{aligned}
$$

and, for $\left(1-z_{1}(\beta f)\right) / \tilde{\delta}^{*} \leq \alpha$,

$$
\begin{aligned}
& \left.\widetilde{\sigma}_{f}\right|_{\text {prepeak }}=g_{1}\left(\phi_{1}\right) 2 \sqrt{\alpha}(1-\alpha) \\
& +g_{1}\left(\phi_{1}\right)\left(\left(\alpha \tilde{\delta}^{*}\right)^{2}-2 \alpha \widetilde{\delta}^{*} \sqrt{\alpha}+\alpha\right)
\end{aligned}
$$

where

$$
g_{1}(\phi)=\frac{1}{4+f^{2}}\left[2+e^{f \phi}(-2 \cos 2 \phi+f \sin 2 \phi)\right]
$$

and

$$
\begin{aligned}
& g_{2}\left(f, \phi_{1}, \phi_{2}\right)=\frac{1}{4+f^{2}}\left[-e^{f \phi_{1}}\left(-2 \cos 2 \phi_{1}+f \sin 2 \phi_{1}\right)\right. \\
& \left.+e^{f \phi_{2}}\left(-2 \cos 2 \phi_{2}+f \sin 2 \phi_{2}\right)\right]
\end{aligned}
$$

And the monotonic bridging constitutive law for the postpeak $\left(\alpha_{f}\right.$ $<1<\alpha)$ is given by, for $\alpha<\left(1-z_{1}\left(\beta_{\jmath}\right)\right) / \widetilde{\delta}^{*}$,

$$
\begin{aligned}
& \left.\widetilde{\sigma}_{f}\right|_{\text {postpeak }}=g_{1}\left(\phi_{1}\right)\left(1-\alpha \widetilde{\delta}^{*}\right)^{2} \\
& +g_{2}\left(f, \phi_{1}, \phi_{2}\right)\left(\left(1-\alpha \widetilde{\delta}^{*}\right)-\left(1-\alpha_{f} \widetilde{\delta}^{*}\right)-\frac{1}{2} \alpha_{f} \beta_{f} \widetilde{\delta}^{*}\right)^{2} \\
& +g_{2}\left(0, \phi_{1}, \phi_{2}\right) 2\left(\left(1-\alpha \widetilde{\delta}^{*}\right)-\left(1-\alpha_{f} \widetilde{\delta}^{*}\right)-\frac{1}{2} \alpha_{f} \beta_{f} \widetilde{\delta}^{*}\right) \frac{1}{n^{*}} \\
& +g_{2}\left(-f, \phi_{1}, \phi_{2}\right)\left(\frac{1}{n^{*}}\right)^{2}
\end{aligned}
$$


and, for $\left(1-z_{1}\left(\beta_{f}\right)\right) / \widetilde{\delta}^{*} \leq \alpha$,

$$
\left.\tilde{\sigma}_{f}\right|_{\text {postpeak }}=g_{1}\left(\phi_{1}\right)\left(1-\alpha \widetilde{\delta}^{*}\right)^{2}
$$

When a fiber composite is fatigue-loaded in the postpeak region $\left(1 \leq \alpha_{f}\right)$ with the crack opening displacement amplitude less than the value for all-fibers-sliding-in $\left(\beta_{f}<\beta_{o}\right)$ and afterwards it is further loaded under monotonic loading, the monotonic constitutive law $\left(1 \leq \alpha_{f}<\alpha\right)$ is given by (19) for $\alpha<\left(1-z_{1}\left(\beta_{f}\right)\right) /$ $\widetilde{\delta}^{*}$ and by $(20)$ for $\left(1-z_{1}\left(\beta_{j}\right)\right) / \widetilde{\delta}^{*} \leq \alpha . \quad \beta_{o}$ is given by

$$
\beta_{o}=\left(\frac{\sqrt{\frac{\widetilde{\delta}_{\max }}{2 \widetilde{\delta}^{*}}}-\sqrt{\frac{\widetilde{\delta}_{\max }}{2 \widetilde{\delta}^{*}}-2 \widetilde{\delta}_{\max }\left(1-\widetilde{\delta}_{\max }\right)}}{\widetilde{\delta}_{\max }}\right)^{2}
$$

When a fiber composite is fatigue-loaded in the postpeak region $\left(1 \leq \alpha_{f}\right)$ with the crack opening displacement amplitude greater than the value for all-fibers-sliding-in $\left(\beta_{0} \leq \beta_{j}\right)$ and afterwards it is further loaded under monotonic loading, the monotonic constitutive law $\left(1 \leq \alpha_{f}<\alpha\right)$ is given by

$$
\begin{aligned}
& \left.\tilde{\sigma}_{f}\right|_{\text {posppeak }}=g_{1}\left(\phi_{5}\right)\left(1-\alpha \widetilde{\delta}^{*}\right)^{2} \\
& +g_{2}\left(f, \phi_{5}, \phi_{2}\right)\left(\left(1-\alpha \widetilde{\delta}^{*}\right)-\left(1-\alpha_{f} \widetilde{\delta}^{*}\right)-\frac{1}{2} \alpha_{f} \beta_{f} \widetilde{\delta}^{*}\right)^{2} \\
& +g_{2}\left(0, \phi_{5}, \phi_{2}\right) 2\left(\left(1-\alpha \widetilde{\delta}^{*}\right)-\left(1-\alpha_{f} \widetilde{\delta}^{*}\right)-\frac{1}{2} \alpha_{f} \beta_{f} \widetilde{\delta}^{*}\right) \frac{1}{n^{*}} \\
& +g_{2}\left(-f, \phi_{5}, \phi_{2}\right)\left(\frac{1}{n^{*}}\right)^{2}
\end{aligned}
$$

It should be noted that for all $\phi_{i}^{\prime} \mathrm{s}$

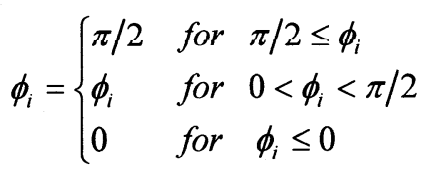

where $i=1 \sim 5$ (Appendix for definitions).

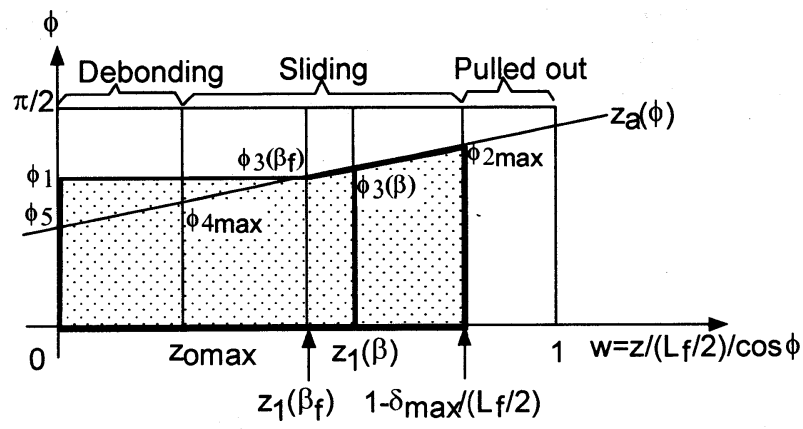

Fig. 8 Domain of the fiber centroidal distance, $\mathrm{z}$, and orientation, $\phi$, for cyclic bridging constitutive law after fiber fatigue rupture

\section{Cyclic Constitutive Law with Fiber Fatigue Rupture}

The cyclic fiber bridging constitutive law with the effect of fiber fatigue rupture is given below in a similar manner to the previous subsection. The domain of surviving fibers is shown in Fig. 8 (see Appendix for details).

When a fiber composite is fatigue-loaded for $n$ cycles in the prepeak region $\left(\alpha_{f} \leq 1\right)$ and afterwards it is loaded under cyclic loading, the cyclic constitutive law is given by, for $\beta \leq \beta$,

$$
\begin{aligned}
& \left.\Delta \widetilde{\sigma}_{f}\right|_{\text {prepeak }}\left(\beta \leq \beta_{f}\right) \\
& =g_{1}\left(\phi_{1}\right) 2 \sqrt{\alpha_{f} \beta}\left(1+\frac{1}{2} \alpha_{f} \beta \widetilde{\delta}^{*}-\alpha_{f} \widetilde{\delta}^{*}-\sqrt{\frac{1}{2} \alpha_{f} \beta}\right) \\
& +g_{2}\left(f, \phi_{1}, \phi_{3}\right) 2 \sqrt{\alpha_{f} \beta}\left(\frac{1}{2} \alpha_{f} \widetilde{\delta}^{*}\left(\beta-\beta_{f}\right)-\sqrt{\frac{1}{2} \alpha_{f} \beta}\right)^{2} \\
& +g_{2}\left(0, \phi_{1}, \phi_{3}\right) 2 \sqrt{\alpha_{f} \beta} \frac{1}{n^{*}} \\
& +g_{1}\left(\phi_{3}\right)\left(\alpha_{f} \beta-\frac{1}{2}\left(\alpha_{f} \beta \widetilde{\delta}^{*}\right)^{2}\right) \\
& +g_{2}\left(f, \phi_{3}, \phi_{2}\right)\left(\alpha_{f} \widetilde{\delta}^{*}\right)^{2}\left(\frac{1}{2} \beta_{f}^{2}-\beta \beta_{f}\right) \\
& +g_{2}\left(0, \phi_{3}, \phi_{2}\right) 2\left(\alpha_{f} \widetilde{\delta}^{*}\right)\left(\beta-\beta_{f}\right) \frac{1}{n^{*}} \\
& +g_{2}\left(-f, \phi_{3}, \phi_{2}\right) 2\left(\frac{1}{n^{*}}\right)^{2}
\end{aligned}
$$

and, for $\beta_{f}<\beta$,

$$
\begin{aligned}
& \left.\Delta \widetilde{\sigma}_{f}\right|_{\text {prepeak }}\left(\beta_{f}<\beta\right) \\
& =g_{1}\left(\phi_{1}\right) 2 \sqrt{\alpha_{f} \beta}\left(1+\frac{1}{2} \alpha_{f} \beta \widetilde{\delta}^{*}-\alpha_{f} \widetilde{\delta}^{*}-\sqrt{\frac{1}{2} \alpha_{f} \beta}\right) \\
& +g_{1}\left(\phi_{1}\right)\left(\alpha_{f} \beta-\frac{1}{2}\left(\alpha_{f} \beta \tilde{\delta}^{*}\right)^{2}\right) \\
& +g_{2}\left(f, \phi_{1}, \phi_{2}\right)\left(\alpha_{f} \widetilde{\delta}^{*}\right)^{2}\left(\frac{1}{2} \beta_{f}^{2}-\beta \beta_{f}\right) \\
& +g_{2}\left(0, \phi_{1}, \phi_{2}\right) 2\left(\alpha_{f} \widetilde{\delta}^{*}\right)\left(\beta-\beta_{f}\right) \frac{1}{n^{*}} \\
& +g_{2}\left(-f, \phi_{1}, \phi_{2}\right) 2\left(\frac{1}{n^{*}}\right)^{2}
\end{aligned}
$$

When a fiber composite is fatigue-loaded for $n$ cycles in the postpeak region $\left(1<\alpha_{f}\right)$ and afterwards it is loaded under cyclic loading $\left(\beta_{f} \leq \beta_{o}\right)$, the cyclic constitutive law is given by (24) for $\beta$ $\leq \beta_{f}$ and by $(25)$ for $\beta$ f $<\beta$.

When a fiber composite is fatigue-loaded for $n$ cycles in the postpeak region $\left(1<\alpha_{f}\right)$ and afterwards it is loaded under cyclic loading $\left(\beta_{o}<\beta_{j}\right)$, the cyclic constitutive law is given by, for $\beta \leq$ 
$\beta$,

$$
\begin{aligned}
& \left.\Delta \widetilde{\sigma}_{f}\right|_{\text {postpeak }}\left(\beta \leq \beta_{f}\right) \\
& =g_{1}\left(\phi_{5}\right) 2 \sqrt{\alpha_{f} \beta}\left(1+\frac{1}{2} \alpha_{f} \beta \widetilde{\delta}^{*}-\alpha_{f} \widetilde{\delta}^{*}-\sqrt{\frac{1}{2} \alpha_{f} \beta}\right) \\
& +g_{2}\left(f, \phi_{5}, \phi_{3}\right) 2 \sqrt{\alpha_{f} \beta}\left(\frac{1}{2} \alpha_{f} \widetilde{\delta}^{*}\left(\beta-\beta_{f}\right)-\sqrt{\frac{1}{2} \alpha_{f} \beta}\right)^{2} \\
& +g_{2}\left(0, \phi_{5}, \phi_{3}\right) 2 \sqrt{\alpha_{f} \beta} \frac{1}{n^{*}} \\
& +g_{1}\left(\phi_{3}\right)\left(\alpha_{f} \beta-\frac{1}{2}\left(\alpha_{f} \beta \widetilde{\delta}^{*}\right)^{2}\right) \\
& +g_{2}\left(f, \phi_{3}, \phi_{2}\right)\left(\alpha_{f} \widetilde{\delta}^{*}\right)^{2}\left(\frac{1}{2} \beta_{f}^{2}-\beta \beta_{f}\right) \\
& +g_{2}\left(0, \phi_{3}, \phi_{2}\right) 2\left(\alpha_{f} \widetilde{\delta}^{*}\right)\left(\beta-\beta_{f}\right) \frac{1}{n^{*}} \\
& +g_{2}\left(-f, \phi_{3}, \phi_{2}\right) 2\left(\frac{1}{n^{*}}\right)^{2}
\end{aligned}
$$

and, for $\beta_{f}<\beta$,

$$
\begin{aligned}
& \left.\Delta \widetilde{\sigma}_{f}\right|_{\text {postpeak }}\left(\beta_{f}<\beta\right) \\
& =g_{1}\left(\phi_{5}\right) 2\left(1-\alpha \widetilde{\delta}^{*}\right)\left(1-\alpha \widetilde{\delta}^{*}+\alpha_{f} \beta \widetilde{\delta}^{*}\right) \\
& +g_{2}\left(f, \phi_{5}, \phi_{2}\right)\left(\alpha_{f} \widetilde{\delta}^{*}\right)^{2}\left(\frac{1}{2} \beta_{f}^{2}-\beta \beta_{f}\right) \\
& +g_{2}\left(0, \phi_{5}, \phi_{2}\right) 2\left(\alpha_{f} \widetilde{\delta}^{*}\right)\left(\beta-\beta_{f}\right) \frac{1}{n^{*}} \\
& +g_{2}\left(-f, \phi_{5}, \phi_{2}\right) 2\left(\frac{1}{n^{*}}\right)^{2}
\end{aligned}
$$

It should be noted that for all $\phi_{i} \mathrm{~s}$

$$
\phi_{i}= \begin{cases}\pi / 2 & \text { for } \pi / 2 \leq \phi_{i} \\ \phi_{i} & \text { for } 0<\phi_{i}<\pi / 2 \\ 0 & \text { for } \quad \phi_{i} \leq 0\end{cases}
$$

where $i=1 \sim 5$ (Appendix for definitions).

\section{Bridging Stress Degradation due to Fiber Fatigue Rupture}

Bridging stress degradation induced by fiber fatigue rupture during fatigue loading is evaluated under monotonic and cyclic loading with the use of the constitutive laws derived above. Fatigue loading can be defined by three parameters: $\alpha$, which is the normalized maximum crack opening displacement in a load cycle, $\beta_{f}$, which is the normalized crack opening displacement amplitude, and $n^{*}$, which is the normalized number of cycles. In the study of crack bridging degradation due to fiber fatigue rupture, fatigue loading means constant crack opening displacementamplitude applied to a cracked fiber composite,
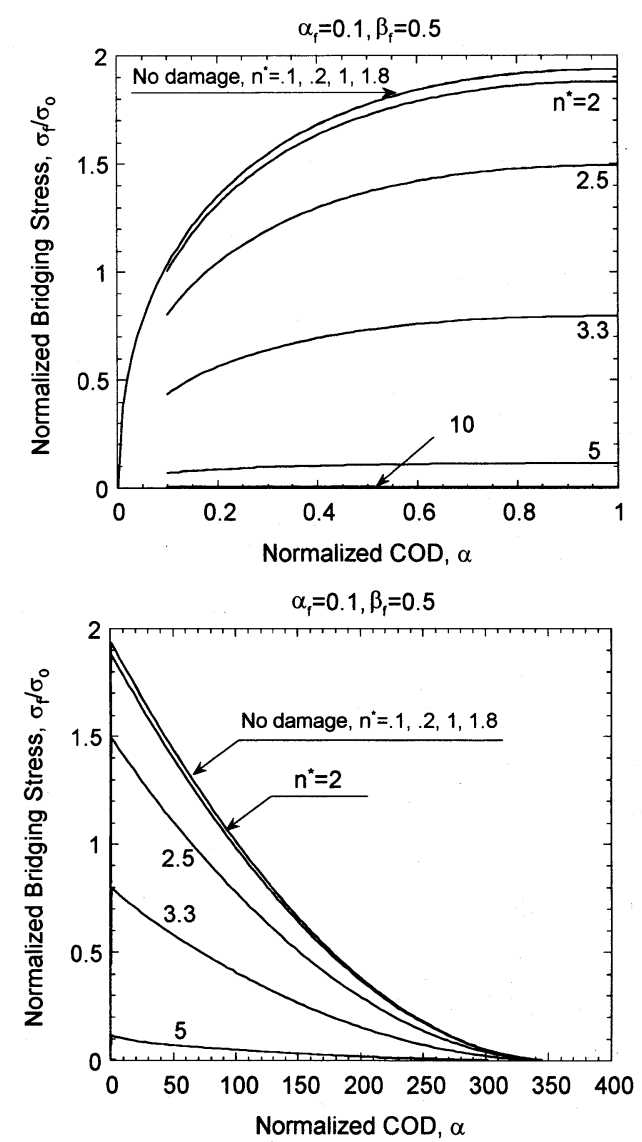

Fig. 9 Monotonic bridging stress degradation $\left(\alpha_{f}=0.1, \beta_{f}=0.5\right)$
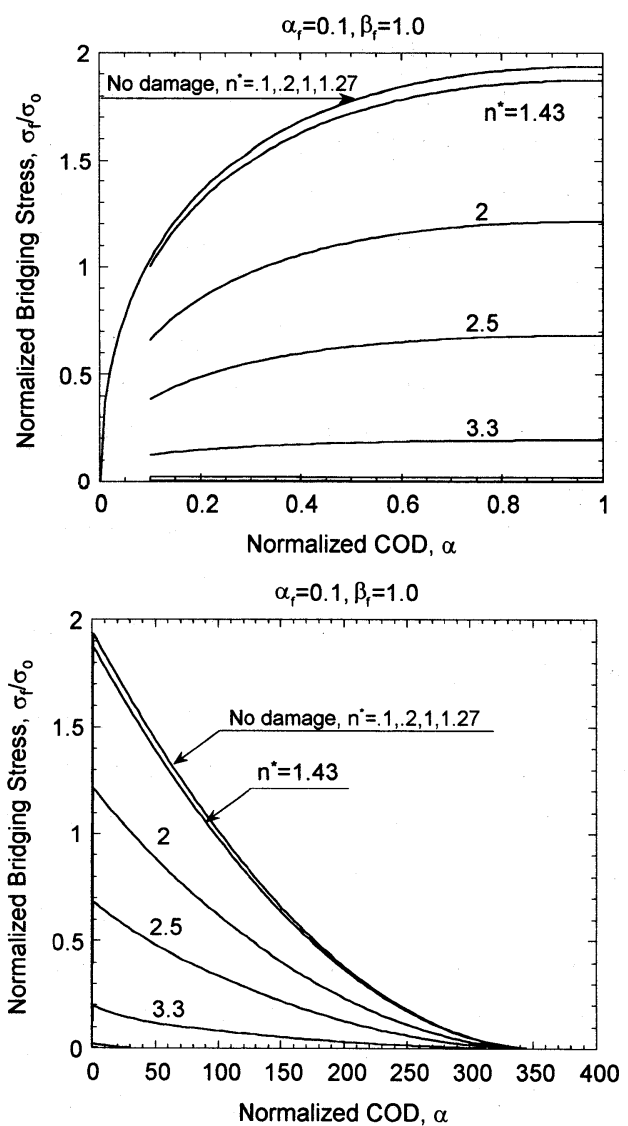

Fig. 10 Monotonic bridging stress degradation $\left(\alpha_{f}=0.1, \beta_{f}=1.0\right)$ 


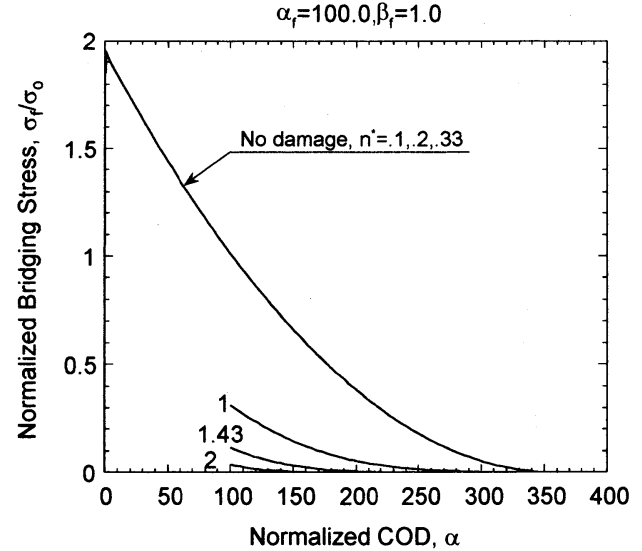

Fig. 11 Monotonic bridging stress degradation $\left(\alpha_{f}=100.0, \beta_{f}=\right.$

1.0)

where $\alpha_{f}$ and $\beta_{f}$ are fixed throughout the fatigue loading. Also, the composite system parameter is set to $\widetilde{\delta}^{*}=0.0028$ for the case of a polyethylene fiber reinforced cementitious composite.

Fig. 9 through Fig. 11 show how the normalized fiber bridging stress, $\tilde{\sigma}_{f}=\sigma_{f} / \sigma_{o}$, changes with the normalized crack opening displacement, $\alpha$, under monotonic loading, after fatigue loading is applied under various conditions $\left(\alpha_{f}=0.1,100.0, \beta_{f}=0.5,1.0\right.$, and $n^{*}=0.1 \sim 10.0$ ). When $\alpha_{f}=0.1$ and $\beta_{f}=0.5$ (half crack closure), the degradation takes place when $n^{*}$ reaches 1.8 (hereafter referred to critical normalized number of cycles, $n_{c r i t}{ }^{*}$ ), and there are no surviving fibers when $n^{*}$ surpasses 10.0. When $\alpha_{f}=0.1$ and $\beta_{f}=1.0$ (full crack closure), the critical value of $n^{*}$ is smaller $\left(n_{c r t}^{*}=1.27\right)$, and the total loss of the bridging stress happens above $n^{*}=5.0$. The difference is due to the crack opening displacement amplitude applied in the two cases. Also, the critical value of $n^{*}$ decreases when $\alpha_{f}$ increases. For $\alpha_{f}=1.0$, $n_{\text {crit }}^{*}$ is $0.57\left(\beta_{f}=0.5\right)$ and $0.4\left(\beta_{f}=1.0\right)$, and, for $\alpha_{f}=100.0, n_{c r i t}^{*}$ is $0.36\left(\beta_{f}=0.5\right)$ and $0.33\left(\beta_{f}=1.0\right)$.

One consequence of fiber fatigue rupture is the decreased bridging strength which appears when $n^{*}$ reaches $n_{c r i t}^{*}$ as exemplified above. Another consequence of fiber fatigue rupture is the decreased crack opening displacement at which the bridging stress vanishes, as seen in Fig. 9 through Fig. 11. Note that at a larger $n^{*}$ the bridging stress vanishes at a smaller $\alpha$ than the original bridging stress, since only fibers with relatively shorter embedment length can survive.

Fig. 12 through Fig. 14 show how the normalized fiber bridging stress amplitude, $\Delta \tilde{\sigma}_{f}=\sigma_{f} / \sigma_{o}$, changes with the normalized crack opening displacement amplitude, $\beta$, under cyclic loading, after fatigue loading is applied under various conditions $\left(\alpha_{f}=0.1\right.$, 100.0, $\beta=0.5,1.0$, and $n^{*}=0.1 \sim 10.0$ ). The trends are the same as in monotonic loading cases. The bridging stress degrades when either $\alpha_{f}, \beta_{f}$, or $n_{c r i t}^{*}$ is increased.

The value of $n_{c r i t}^{*}$ is the same for both of monotonic and cyclic loading case, since the monotonic and cyclic bridging stresses are exerted by the same surviving fibers in the reduced

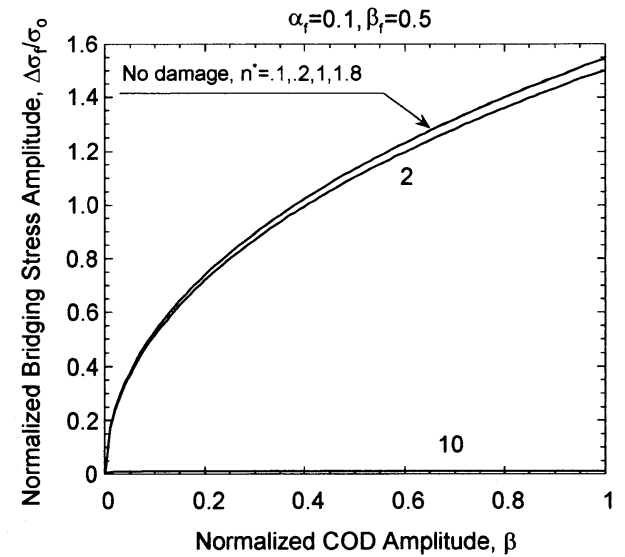

Fig. 12 Cyclic bridging stress degradation $\left(\alpha_{f}=0.1, \beta_{f}=0.5\right)$

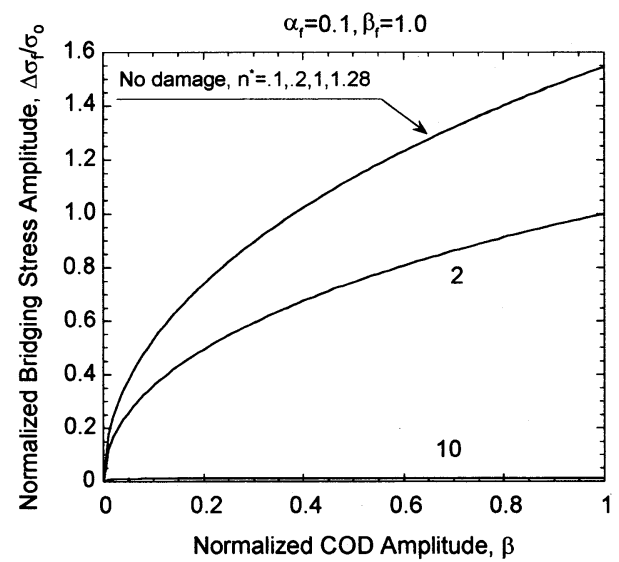

Fig. 13 Cyclic bridging stress degradation $\left(\alpha_{f}=0.1, \beta_{f}=1.0\right)$

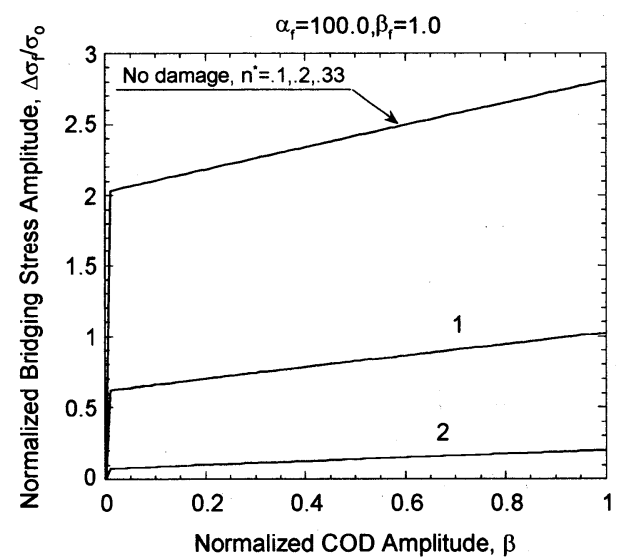

Fig. 14 Cyclic bridging stress degradation $\left(\alpha_{f}=100.0, \beta_{f}=1.0\right)$

domain. Indeed, $n_{c r i t}^{*}$ can be readily obtained (Appendix). When fatigue loading is applied in the prepeak $\left(\alpha_{f}<1\right)$ or when fatigue loading is applied in the postpeak $\left(\alpha_{f}>1\right)$ and its amplitude is small $\left(\beta_{f}<\beta_{o}\right), n_{\text {crit }}^{*}$ is given by, in a normalized form,

$$
n_{c r i t}^{*}=e^{-f \frac{\pi}{2}}\left(\sqrt{\frac{\alpha_{f} \beta_{f}}{2}}\right)^{-1}
$$

or 


$$
n_{c r i t}^{*}=e^{-f \frac{\pi}{2}}\left(\sqrt{\frac{1}{2} \frac{\Delta \widetilde{\delta}}{\widetilde{\delta}^{*}}}\right)^{-1}
$$

When fatigue loading is applied in the postpeak $\left(\alpha_{p}>1\right)$ and its amplitude is large $\left(\beta_{o}<\beta_{j}\right), n_{c r i t}^{*}$ is given by, in a normalized form,

$$
n_{c r i t}^{*}=e^{-f \frac{\pi}{2}}\left(\frac{\alpha_{f} \beta_{f} \widetilde{\delta}^{*}}{2}+1-\alpha_{f} \widetilde{\delta}^{*}\right)^{-1}
$$

or

$$
n_{c r i t}^{*}=e^{-f \frac{\pi}{2}}\left(\frac{1}{2} \Delta \widetilde{\delta}+1-\widetilde{\delta}_{\max }\right)^{-1}
$$

As shown above, the degradation of fiber bridging stresses starts when $n^{*}$ exceeds $n_{c r i t}^{*}$. The two equations above are the condition for a single fiber inclined at 90 degrees to the crack plane to be fatigue-ruptured and are for a fiber in debonding and sliding respectively. This $n_{c r i t}^{*}$ tells the point at which fibers start to be ruptured not only under constant crack opening displacement amplitude condition, but also under constant stress amplitude condition, since two conditions are the same until load redistribution takes place upon the rupture of the first fiber. However, when $n^{*}>n_{c r i t}^{*}$ under constant stress amplitude condition, bridging stress curves are different from those under constant crack opening displacement amplitude condition. Also, it should be noted that, under variable stress amplitude condition, $n_{c r i t}^{*}$ does not hold valid.

\section{Concluding Remarks}

This paper presented a theoretical formulation of the cyclic constitutive law for a discontinuous fiber reinforced composite with the effects of fiber fatigue rupture. The formulation is based on the micromechanics of fiber bridging under cyclic loading, enabling the effects of microstructural parameters to be evaluated.

The single fiber behavior ( $P$ - $\delta$ and $\Delta P-\Delta \delta$ relation) was reviewed. Also, the notations of fiber bridging constitutive law under cyclic loading $\left(\Delta \sigma_{f}-\Delta \delta\right)$ were introduced based on the previous studies.

Effects of fiber fatigue rupture are included in the constitutive laws under monotonic and cyclic loading, accounting for the fatigue rupture of randomly distributed fibers under constant crack opening displacement amplitude. As fiber fatigue rupture proceeds, maximum bridging stress as well as crack opening displacement at which bridging stress vanishes are decreased. The critical normalized number of cycles, $n_{c r i t}^{*}$ at which crack bridging degradation takes place, is analytically derived.

For the future tasks, it is necessary to conduct experimental measurements to show the validity of the derived fiber bridging constitutive law, to apply the fiber bridging constitutive law to progressive crack occurrence and growth in a structural analysis, and also to extend the constitutive law beyond the assumptions of frictional bond controlled interface and no fiber rupture under static loading.

\section{Appendix Derivation of the Monotonic and Cyclic Fiber Bridging Constitutive Laws with Effect of Fiber Fatigue Rupture}

The monotonic and cyclic fiber bridging constitutive laws with effect of fiber fatigue rupture can be derived in a similar way to the cyclic fiber bridging constitutive law derived by Matsumoto ${ }^{1}$. The difference is the integration domain reduced due to progressive fiber fatigue rupture (Fig. 7 for monotonic and Fig. 8 for cyclic constitutive law). $\phi_{1}$ 's $(i=1 \sim 5)$ defined in Fig. 7 and Fig. 8 determine the integration domain and have expressions as follows:

$$
\begin{aligned}
& \phi_{1}=\frac{1}{f} \ln \left[\frac{1}{n^{c}} \frac{L_{c}}{L_{f}}\left(\sqrt{\frac{1}{2} \alpha_{f} \beta_{f}}\right)^{-1}\right] \\
& \phi_{2 \max }=\frac{1}{f} \ln \left[\frac{1}{n^{c}} \frac{L_{c}}{L_{f}}\left(\frac{1}{2} \alpha_{f} \beta_{f} \widetilde{\delta}^{*}\right)^{-1}\right] \\
& \phi_{2}=\frac{1}{f} \ln \left[\frac{1}{n^{c}} \frac{L_{c}}{L_{f}}\left(\frac{1}{2} \alpha_{f} \beta_{f} \widetilde{\delta}^{*}-\alpha_{f} \widetilde{\delta}^{*}+\alpha \widetilde{\delta}^{*}\right)^{-1}\right]
\end{aligned}
$$

$$
\phi_{3}(\beta)=\frac{1}{f} \ln \left[\frac{1}{n^{c}} \frac{L_{c}}{L_{f}}\left(\frac{1}{2} \alpha_{f} \widetilde{\delta}^{*}\left(\beta_{f}-\beta\right)+\sqrt{\frac{1}{2} \alpha_{f} \beta}\right)^{-1}\right]
$$

$$
\phi_{3}\left(\beta_{f}\right)=\phi_{1}
$$

$$
\phi_{4 \max }=\frac{1}{f} \ln \left[\frac{1}{n^{c}} \frac{L_{c}}{L_{f}}\left(\frac{1}{2} \alpha_{f} \beta_{f} \tilde{\delta}^{*}-\alpha_{f} \tilde{\delta}^{*}+\sqrt{\alpha_{f}}\right)^{-1}\right]
$$

$$
\phi_{4}=\frac{1}{f} \ln \left[\frac{1}{n^{c}} \frac{L_{c}}{L_{f}}\left(\frac{1}{2} \alpha_{f} \beta_{f} \widetilde{\delta}^{*}-\alpha_{f} \widetilde{\delta}^{*}+\sqrt{\alpha}\right)^{-1}\right]
$$

and

$$
\phi_{5}=\frac{1}{f} \ln \left[\frac{1}{n^{c}} \frac{L_{c}}{L_{f}}\left(\frac{1}{2} \alpha_{f} \beta_{f} \widetilde{\delta}^{*}+1-\alpha_{f} \widetilde{\delta}^{*}\right)^{-1}\right]
$$

It should be noted that, for all $\phi_{1}$ 's in the equations below, 


$$
\phi_{i}=\left\{\begin{array}{lll}
\pi / 2 & \text { for } & \pi / 2 \leq \phi_{i} \\
\phi_{i} & \text { for } & 0<\phi_{i}<\pi / 2 \\
0 & \text { for } & \phi_{i} \leq 0
\end{array}\right.
$$

where $i=1 \sim 5$.

\section{Monotonic Constitutive Law}

The monotonic constitutive law after fiber fatigue rupture can be obtained by integrating the contribution of surviving fibers in the integration domain of $w$ and $\phi$ shown in Fig. 7. The surviving fibers again divided into two groups: fibers in debonding and sliding. As the bridged crack opens up under monotonic loading, fibers will undergo debonding, sliding, and pulled-out. Only fibers in debonding and sliding contribute to the bridging stress. The relation between the fiber pull-out load, $P$, and the crack opening displacement, $\delta$, of a single fiber in debonding is given by (1) and the relation for a single fiber in sliding is given by (2).

When a fiber composite is fatigue-loaded for $n$ cycles in the prepeak region $\left(\alpha_{f}=\widetilde{\delta}_{\max } / \widetilde{\delta}^{*}<1\right)$ and afterwards it is further loaded under monotonic loading, the monotonic constitutive law for the prepeak $\left(\alpha_{f}<\alpha=\widetilde{\delta} / \widetilde{\delta}^{*}<1\right)$ can be obtained by, for $\alpha$ $<\left(1-z_{1}\left(\beta_{f}\right)\right) / \widetilde{\delta}^{*}$,

$$
\begin{aligned}
& \left.\tilde{\sigma}_{f}\right|_{\text {prepeak }}=\frac{8}{\pi \tau\left(L_{f} / d_{f}\right) d_{f}^{2}}\left\{\int_{\phi=0}^{\phi_{1}} \int_{z^{\prime}=0}^{z_{o} \cos \phi} P_{d}(\delta) e^{f \phi} \sin \phi d z^{\prime} d \phi\right. \\
& +\int_{\phi=0}^{\phi_{1}} \int_{z^{\prime}=z_{o} \cos \phi}^{\left(1-\tilde{\delta}_{\max }\right) \cos \phi} P_{s l}(\delta) e^{f \phi} \sin \phi d z^{\prime} d \phi \\
& \left.+\int_{\phi-\phi_{1} z^{\prime}-z_{a}(\phi) \cos \phi}^{\phi_{2}} \int_{\left(1-\widetilde{\delta}_{\max }\right) \cos \phi}^{\left(c_{s l}\right.}(\delta) e^{f \phi} \sin \phi d z^{\prime} d \phi\right\}
\end{aligned}
$$

which reduces to (15), and, for $\left(1-z_{1}\left(\beta_{f}\right)\right) / \widetilde{\delta}^{*} \leq \alpha$,

$$
\begin{aligned}
& \left.\tilde{\sigma}_{f}\right|_{\text {prepeak }}=\frac{8}{\pi \tau\left(L_{f} / d_{f}\right) d_{f}^{2}}\left\{\int_{\phi=0}^{\phi_{1}} \int_{z^{\prime}=0}^{z_{o}} P_{d}(\delta) e^{f \phi} \sin \phi d z^{\prime} d \phi\right. \\
& \left.+\int_{\phi=0}^{\phi_{1}} \int_{z^{\prime}=z_{o} \cos \phi}^{\left(1-\tilde{\delta}_{\text {max }}\right) \cos \phi} P_{s l}(\delta) e^{f \phi} \sin \phi d z^{\prime} d \phi\right\}
\end{aligned}
$$

which reduces to (16). And the monotonic bridging constitutive law for the postpeak $\left(\alpha_{f}<1<\alpha\right)$ can be obtained by, for $\alpha<(1$ $\left.z_{1}\left(\beta_{f}\right)\right) / \widetilde{\delta}^{*}$,

$$
\begin{aligned}
& \left.\tilde{\sigma}_{f}\right|_{\text {postpeak }}=\frac{8}{\pi \tau\left(L_{f} / d_{f}\right) d_{f}^{2}}\left\{\int_{\phi=0}^{\phi_{1}\left(1-\tilde{\delta}_{\text {max }}\right) \cos \phi} \int_{z^{\prime}=0}^{1} P_{s l}(\delta) e^{f \phi} \sin \phi d z^{\prime} d \phi\right. \\
& \left.+\int_{\phi=\phi_{1}}^{\phi_{2}} \int_{z^{\prime}=z_{a}(\phi) \cos \phi}^{\left(1-\tilde{\delta}_{\max } \cos \phi\right.} P_{s l}(\delta) e^{f \phi} \sin \phi d z^{\prime} d \phi\right\}
\end{aligned}
$$

which reduces to (19), and, for $\left(1-z_{1}\left(\beta_{f}\right)\right) / \widetilde{\delta}^{*} \leq \alpha$,

$$
\left.\tilde{\sigma}_{f}\right|_{\text {postpeak }}=\frac{8}{\pi \tau\left(L_{f} / d_{f}\right) d_{f}^{2}}\left\{\int_{\phi=0}^{\phi_{1}} \int_{z^{\prime}=0}^{\left(1-\tilde{\delta}_{\text {max }}\right) \cos \phi} P_{s l}(\delta) e^{f \phi} \sin \phi d z^{\prime} d \phi\right\}
$$

which reduces to (20).

When a fiber composite is fatigue-loaded in the postpeak region $\left(1 \leq \alpha_{f}\right)$ with the crack opening displacement amplitude less than the value for all-fibers-sliding-in $\left(\beta_{f}<\beta_{o}\right)$ and afterwards it is further loaded under monotonic loading, the monotonic constitutive law $\left(1 \leq \alpha_{f}<\alpha\right)$ can be obtained by (A12) for $\alpha<$ (1 $\left.-z_{1}\left(\beta_{f}\right)\right) / \widetilde{\delta}^{*}$ and by (A13) for $\left(1-z_{1}\left(\beta_{f}\right)\right) / \widetilde{\delta}^{*} \leq \alpha$.

When a fiber composite is fatigue-loaded in the postpeak region $\left(1 \leq \alpha_{f}\right)$ with the crack opening displacement amplitude greater than the value for all-fibers-sliding-in $\left(\beta_{o} \leq \beta_{f}\right)$ and afterwards it is further loaded under monotonic loading, the monotonic constitutive law $\left(1 \leq \alpha_{f}<\alpha\right)$ can be obtained by

$$
\begin{aligned}
& \left.\tilde{\sigma}_{f}\right|_{\text {postpeak }}=\frac{8}{\pi \tau\left(L_{f} / d_{f}\right) d_{f}^{2}}\left\{\int_{\phi=0}^{\phi_{s}\left(1-\tilde{\delta}_{\text {max }}\right) \cos \phi} \int_{z^{\prime}=0} P_{s l}(\delta) e^{f \phi} \sin \phi d z^{\prime} d \phi\right. \\
& \left.+\int_{\phi=\phi_{5} z^{\prime}=z_{a}(\phi) \cos \phi}^{\phi_{2}\left(1-\tilde{\delta}_{\text {max }}\right) \cos \phi} P_{s l}(\delta) e^{f \phi} \sin \phi d z^{\prime} d \phi\right\}
\end{aligned}
$$

which reduces to (22).

\section{Cyclic Constitutive Law}

The cyclic constitutive law after fiber fatigue rupture can be obtained in a similar way to the monotonic law above. The surviving fibers are divided into two groups: fibers in unstretching and contracting and in sliding-in, and the division is made by $z_{1}(\beta)$ in Fig. 8. All surviving fibers contribute to the bridging stress, since none of them will be discounted (no pull-out in cyclic loading).

When a fiber composite is fatigue-loaded for $n$ cycles in the prepeak region $\left(\alpha_{f}<1\right)$ and afterwards it is loaded under cyclic loading, the cyclic constitutive law can be obtained by, for $\beta \leq \beta_{\text {, }}$, 


$$
\begin{aligned}
& \left.\Delta \tilde{\sigma}_{f}\right|_{\text {prepeak }}\left(\beta \leq \beta_{f}\right)=\frac{8}{\pi \tau\left(L_{f} / d_{f}\right) d_{f}^{2}} \\
& \left\{\int_{\phi=0}^{\phi_{1}} \int_{z^{\prime}=0}^{z_{1}(\beta) \cos \phi} \Delta P_{1}(\Delta \delta) e^{f \phi} \sin \phi d z^{\prime} d \phi\right. \\
& +\int_{\phi=\phi_{1}}^{\phi_{3}(\beta)=z_{a}(\phi) \cos \phi} \int_{z_{1}(\beta) \cos \phi}^{z_{\phi}} \Delta P_{1}(\Delta \delta) e^{f \phi} \sin \phi d z^{\prime} d \phi \\
& +\int_{\phi=0}^{\phi_{3}} \int_{z^{\prime}=z_{1}(\beta) \cos \phi}^{\left(1-\widetilde{\delta}_{\max }\right) \cos \phi} \Delta P_{3}(\Delta \delta) e^{f \phi} \sin \phi d z^{\prime} d \phi
\end{aligned}
$$

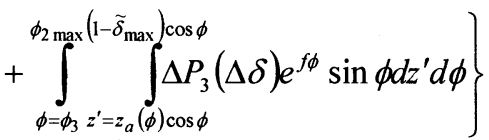

which reduces to (24), and, for $\beta_{f}<\beta$,

$$
\begin{aligned}
& \left.\Delta \widetilde{\sigma}_{f}\right|_{\text {prepeak }}\left(\beta_{f}<\beta\right)=\frac{8}{\pi \tau\left(L_{f} / d_{f}\right) d_{f}^{2}} \\
& \left\{\int_{\phi=0}^{\phi_{1} z_{1}(\beta) \cos \phi} \int_{z^{\prime}=0}^{z_{1}} \Delta P_{1}(\Delta \delta) e^{f \phi} \sin \phi d z^{\prime} d \phi\right. \\
& +\int_{\phi=0}^{\phi_{1}} \int_{z^{\prime}=z_{1}(\beta) \cos \phi}^{\left(1-\tilde{\delta}_{\text {max }}\right) \cos \phi} \Delta P_{3}(\Delta \delta) e^{f \phi} \sin \phi d z^{\prime} d \phi \\
& \left.+\int_{\phi=\phi_{1} z^{\prime}=z_{a}(\phi) \cos \phi}^{\phi_{2 \max }\left(1-\tilde{\delta}_{\text {max }} \cos \phi\right.} \Delta P_{3}(\Delta \delta) e^{f \phi} \sin \phi d z^{\prime} d \phi\right\}
\end{aligned}
$$

which reduces to (25).

When a fiber composite is fatigue-loaded for $n$ cycles in the postpeak region $\left(1<\alpha_{f}\right)$ and afterwards it is loaded under cyclic loading $\left(\beta_{f}<\beta_{o}\right)$, the cyclic constitutive law can be obtained by (A15) for $\beta \leq \beta_{f}$ and by (A16) for $\beta<\beta$.

When a fiber composite is fatigue-loaded for $n$ cycles in the postpeak region $\left(1<\alpha_{f}\right)$ and afterwards it is loaded under cyclic loading $\left(\beta_{o}<\beta_{j}\right)$, the cyclic constitutive law can be obtained by, for $\beta \leq \beta$,

$$
\begin{aligned}
& \left.\Delta \tilde{\sigma}_{f}\right|_{\text {postpeak }}\left(\beta \leq \beta_{f}\right)=\frac{8}{\pi \tau\left(L_{f} / d_{f}\right) d_{f}^{2}} \\
& \left\{\int_{\phi=0}^{\phi_{5}} \int_{z^{\prime}=0}^{z_{1}(\beta) \cos \phi} \Delta P_{1}(\Delta \delta) e^{f \phi} \sin \phi d z^{\prime} d \phi\right.
\end{aligned}
$$

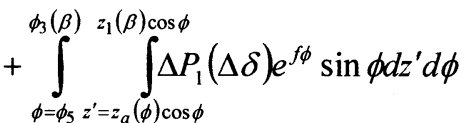

$$
\begin{aligned}
& +\int_{\phi=0}^{\phi_{3}} \int_{z^{\prime}=z_{1}(\tilde{\beta}) \cos \phi}^{\left(1-\tilde{\delta}_{\max }\right) \cos \phi} \Delta P_{3}(\Delta \delta) e^{f \phi} \sin \phi d z^{\prime} d \phi \\
& \left.+\int_{\phi=\phi_{3}}^{\phi_{2}=z_{a}(\phi) \cos \phi} \int_{\left(1-\widetilde{\delta}_{\max }\right) \cos \phi} \Delta P_{3}(\Delta \delta) e^{f \phi} \sin \phi d z^{\prime} d \phi\right\}
\end{aligned}
$$

which reduces to (26) and, for $\beta<\beta$,

$$
\begin{aligned}
& \left.\Delta \tilde{\sigma}_{f}\right|_{\text {pospeak }}\left(\beta_{f}<\beta\right)=\frac{8}{\pi \tau\left(L_{f} / d_{f}\right) d_{f}^{2}} \\
& \left\{\int_{\phi=0}^{\phi_{5}\left(1-\tilde{\delta}_{\max }\right) \cos \phi} \int_{z^{\prime}=0} \Delta P_{3}(\Delta \delta) e^{f \phi} \sin \phi d z^{\prime} d \phi\right. \\
& \left.+\int_{\phi=\phi_{5} z^{\prime}=z_{a}(\phi) \cos \phi}^{\phi_{2 \max }\left(1-\tilde{\delta}_{\max }\right) \cos \phi} \Delta P_{3}(\Delta \delta) e^{f \phi} \sin \phi d z^{\prime} d \phi\right\}
\end{aligned}
$$

which reduces to (27).

\section{References}

1) Matsumoto, T., Crack Bridging Law in Discontinuous Fiber Reinforced Composites under Cyclic Loading, Journal of Applied Mechanics, 10, 923-933, 2007.

2) Evans, A. G. (1997). "Design and Life Prediction Issues for High-Temperature Engineering Ceramics and Their Composites." Acta Materialia, 45(1), 23-40.

3) Evans, A. G., Zok, F. W., and McMeeking, R. M. (1995). "Fatigue of Ceramic Matrix Composites." Acta Metallurgica et Materialia, 43(3), 859-875.

4) Zok, F. W., McNulty, J., Du, Z. Z., and Evans, A. G. (1994). "Effects of Interfacial Wear on Fatigue Failure in Fiber Reinforced CMCs." International Conference on Composites Engineering-ICCE/1,597-598.

5) Cho, C., Holmes, J. W., and Barber, J. R. (1991). "Estimation of Interfacial Shear in Ceramic Composites from Frictional Heating Measurements." Journal of the American Ceramic Society, 74(11), 2802-2808.

6) Holmes, J. W., and Cho, C. (1992). "Experimental Observations of Frictional Heating in Fiber-Reinforced Ceramics." Journal of the American Ceramic Society, 75(4), 929-938.

7) Dambrine, B. (1994). "Low Cycle Fatigue of $\mathrm{SiC} / \mathrm{SiC}$ Composites at High Temperature - Role of the Oxidation of the Interface on the Cyclic Behaviour and Modelling." International Conference on Composites Engineering-ICCE/1, 679-680.

8) Rouby, D., and Reynaud, P. (1993). "Fatigue Behavior Related to Interface Modification during Load Cycling in Ceramic-Matrix Fibre Composites." Composites Science and Technology, 48, 109-118.

9) Zhang, J., Stang, H., and Li, V. C., Experimental Study on Crack Bridging in FRC under Uniaxial Fatigue Tension, Journal of Materials in Civil Engineering, 12(1), 66-73, 2000.

10) Li, V. C. and Matsumoto, T., Fatigue Crack Growth Analysis of Fiber Reinforced Concrete with Effect of Interfacial Bond Degradation, Journal of Cement and Concrete Composites, 
20(5), 339-351, 1998.

11) Matsumoto, T. and Li, V. C., Fatigue Life Analysis of Fiber Reinforced Concrete with a Fracture Mechanics Based Model, Journal of Cement and Concrete Composites, 21(4), 249-261, 1999.

12) Matsumoto, T. and Li, V. C., Fracture Mechanics Based Fatigue Life Model of Discontinuous Fiber Reinforced Composites, Proceedings of the Japan Concrete Institute, 20(3), 163-168, 1998. (in Japanese)

13) Walls, D. P., Bao, G., and Zok, F. W., Mode I Fatigue Cracking in a Fiber Reinforced Metal Matrix Composite, Acta Metallurgica et Materialia, 41(7), 2061-2071, 1993.

14) Matsumoto, T., Fracture Mechanics Approach to Fatigue Life of Discontinuous Fiber Reinforced Composites, Doctoral Thesis, The University of Michigan, Ann Arbor. 1998.

15) Suthiwarapirak, P., Matsumoto, T., and Kanda, T., Flexural Fatigue Failure Characteristics of an Engineered Cementitious Composite and Polymer Cement Mortars, Journal of Materials, Concrete Structures and Pavements, No. 718/V-57, 121-134, 2002.
16) Suthiwarapirak, P., Matsumoto, T., and Kanda, T., Multiple Cracking and Fiber Bridging Characteristics of Engineered Cementitious Composites under Fatigue Flexure, Journal of Materials in Civil Engineering, 16(5), 433-443, 2004.

17) Matsumoto, T. and Li, V. C., Uniaxial Cyclic Behavior of Discontinuous Fiber Reinforced Composites. Proc. ASCE 4th Materials Engineering Conference, Washington D.C., USA, 426-435, 1996.

18) Li, V. C., Postcrack Scaling Relations for Fiber Reinforced Cementitious Composites, Journal of Materials in Civil Engineering, 4(1), 41-57, 1992.

19) Li, V. C., Wang, Y., and Backer, S., Effect of Inclining Angle, Bundling, and Surface Treatment on Synthetic Fiber Pull-Out from a Cement Matrix, Journal of Composites, 21(2), 132-140. 1990.

(Received: April 14, 2008) 